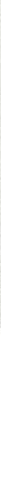

\title{
Análise dos principais modelos de indicadores para cidades sustentáveis e inteligentes
}

Analysis of key indicator models for sustainable and smart cities

Análisis de los principales modelos de indicadores para ciudades sostenibles e inteligentes

\author{
Luciana Regina Bencke \\ Mestranda, PPGTIC-UFSC \\ luciana.bencke@gmail.com
}

\section{Anderson Luiz Fernandes Perez \\ Doutor, PPGTIC-UFSC anderson.perez@ufsc.br}




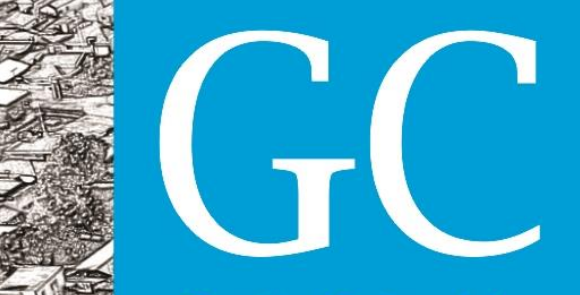

Revista Nacional de

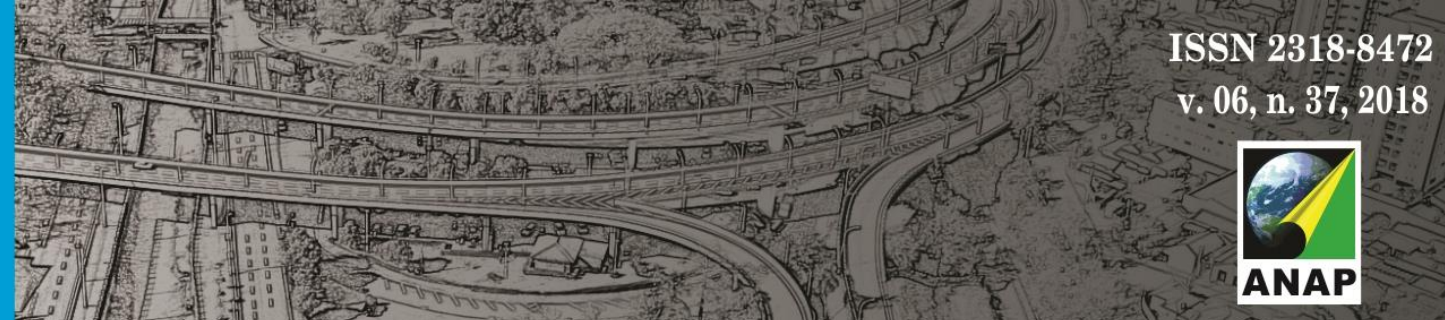

Gerenciamento de Cidades

\section{RESUMO}

A utilização dos rankings de cidades é uma forma de identificar os pontos fortes e fracos do município. Esta prática vem se acentuando na última década com vários rankings ligados ao conceito de cidades inteligentes e sustentáveis. A comparação com outras cidades propicia maior conscientização dos atores da cidade sobre o meio urbano: gestores públicos, cidadãos e empresas. Entretanto, é necessário atentar para particularidades de cada localidade e analisar modelos de indicadores existentes contribui no desenvolvimento do conjunto de indicadores certos e na aplicação do modelo mais aderente à realidade local. O método de análise utilizado nesta pesquisa pode ser aplicado a outros modelos e permite o avanço em pesquisas relacionadas ao gerenciamento municipal alinhado ao recente conceito de cidades inteligentes e sustentáveis. Além disso, essa pesquisa pode apoiar gestores públicos e demais atores da cidade fornecendo uma síntese dos modelos estudados, com uma análise comparativa das dimensões abordadas em cada um, objetivos, público alvo, escopo, metodologia e meios de disseminação dos resultados.

PALAVRAS-CHAVE: Indicadores Urbanos. Ranking das Cidades. Cidades Inteligentes. Cidades Sustentáveis.

\section{ABSTRACT}

The use of city rankings helps identifying the strengths and weaknesses of the municipality. This practice has been accentuated in the last decade with several rankings linked to the concept of smart and sustainable cities. The comparison with other cities provides a greater awareness of the city's actors on the urban environment: public managers, citizens and companies. However, it is necessary to look at particularities of each locality and analyzing existing indicators models contributes to the development of the set of certain indicators and to apply the more adherent model for the local reality. The analysis method used in this research can be applied to other models and allows advancement in research related to municipal management in line with the recent concept of intelligent and sustainable cities. In addition, this research can support public managers and other city's actors providing a synthesis of the studied models, with a comparative analysis of the dimensions addressed in each one, objectives, target audience, scope, methodology and means of dissemination of results.

KEY WORDS: Urban Indicators. City Ranking. Smart Cities. Sustainable Cities.

\section{RESUMEN}

La utilización de los rankings de ciudades es una forma de identificar los puntos fuertes y frágiles del municipio. Esta práctica se ha acentuado en la última década con varios rankings ligados al concepto de ciudades inteligentes y sostenibles. La comparación con otras ciudades propicia mayor concientización de los actores de la ciudad sobre el medio urbano: gestores públicos, ciudadanos y empresas. Sin embargo, es necesario atentar para particularidades de cada localidad y analizar modelos de indicadores existentes contribuye en el desarrollo del conjunto de indicadores adecuados y en la aplicación del modelo más adherente a la realidad local. El método de análisis utilizado en esta investigación puede ser aplicado a otros modelos y permite el avance en investigaciones relacionadas con la gestión municipal alineado al reciente concepto de ciudades inteligentes y sostenibles. Además, esta investigación puede apoyar a gestores públicos y demás actores de la ciudad proporcionando una síntesis de los modelos estudiados, con un análisis comparativo de las dimensiones abordadas en cada uno, objetivos, público, alcance, metodología y medios de diseminación de los resultados.

PALABRAS CLAVE: Indicadores urbanos. Ranking de ciudades. Ciudades inteligentes. Ciudades Sostenibles. 


\section{INTRODUÇÃO}

Indicadores econômicos e sociais, como taxa de desemprego, produto interno bruto (PIB), índices de preços e inflação são utilizados por governos na avaliação da performance das nações desde o início do século 20. Após a segunda guerra agências internacionais como Organização Mundial da Saúde (WHO), a Organização para Cooperação e Desenvolvimento Econômico (OECD - Organisation for Economic Co-operation and Development) e o Programa de Desenvolvimento das Nações Unidas (UNDP - United Nations Development Programme) se dedicam a medir, classificar e rastrear o desempenho e a produtividade de vários fenômenos de saúde, econômicos e sociais em todas as nações e regiões [KITCHIN et. Al, 2015]. Em setembro de 2015, a Organização das Nações Unidas (ONU) propôs 17 ODS (Objetivos de Desenvolvimento da Sustentabilidade) divididos em 169 metas, onde o objetivo 11 se refere a transformar as cidades e comunidades em ambientes inclusivos, seguros, resilientes e sustentáveis [ONU, 2015]. Este objetivo possui 10 metas associadas as quais [BHATTACHARYA et. al, 2016] resumem em 4 grupos: (1) Habitação e transporte adequados, seguros e acessíveis a todos os cidadãos em todas as categorias de idade, gênero, poder aquisitivo e outras categorias marginais e vulneráveis; (2) Impacto mínimo das catástrofes (óbitos e perdas econômicas), impacto ambiental adverso e mudanças climáticas; (3) Proteção do patrimônio cultural e natural, uso de material local; e (4) Capacitação para fazer os 3 pontos anteriores e para implementar planejamento participativo. Incentivar as cidades a criar um sistema de indicadores que se conecte a objetivos locais de sustentabilidade e inteligência tecnológica tem sido uma abordagem em várias regiões que buscam uma gestão pública mais efetiva. [KAPLAN e NORTON, 2000] ressaltam que o que você mede é o que você obtém, pois o que você pode medir é o que você pode gerenciar. Para obter avanços significativos no desempenho são necessárias grandes mudanças, e isso inclui mudanças nos sistemas de medição e gerenciamento usados por uma organização. Os autores reforçam que para um futuro cada vez mais competitivo, orientado à tecnologia e à competência, as organizações não podem realizar a medição do desempenho somente através da monitoração e controle somente de medidas que retratam o passado, é fundamental que as informações sejam atualizadas o máximo possível. Uma cidade é também uma organização a qual se aplica a reflexão de Kaplan e Norton, pois a utilização de indicadores é primordial no processo decisório, desde a escolha das iniciativas corretas a priorizar até o acompanhamento dos resultados das mesmas de forma a transformar o ambiente urbano em locais sustentáveis e com uma boa qualidade de vida para os cidadãos.

A busca por desenvolver cidades sustentáveis e inteligentes em todo o mundo vem como resposta a um movimento acelerado de urbanização que se iniciou a algumas décadas. Em 2014 54\% da população mundial residia em áreas urbanas, e a previsão é que esse movimento de urbanização continue atingindo quase $70 \%$ em 2050 [ONU, 2014]. No Brasil, o censo de 2010 já registrou 83\% da população residente nas cidades [RBClH, 2016]. Esta transformação traz uma série de desafios urbanos e fazer uma cidade "inteligente" está emergindo como uma estratégia para mitigar os problemas gerados pela rápida urbanização. Neste sentido, uma Smart City (Cidade Inteligente em inglês) pode ser definida como uma cidade de alta tecnologia com várias capacidades para resolução de problemas [Sharma \& Rajput, 2017]. O foco da solução de problemas também é abordado na visão na ISO (International Organization for Standardization): 


\begin{abstract}
A "inteligência" de uma cidade está relacionada a competência de reunir seus recursos para cumprir, de forma efetiva e transparente, os propósitos estabelecidos através da aplicação de uma nova geração de tecnologias da informação, como a internet das coisas, computação em nuvem, big data, integração de informações geográficas / espaciais, as quais facilitam o planejamento, a implementação e o gerenciamento dos serviços das cidades [ISO, 2014a].
\end{abstract}

Sistemas de indicadores em processos de governança com muitos atores, como é o caso das cidades, facilitam a abertura do diálogo, possibilitando o compartilhamento de informações, aprendizado e consenso entre especialistas e leigos, entre governo formal, empresas e cidadãos, e entre diferentes níveis governos (federal, estadual ou municipal) [HOLDEN, 2012]. Desta forma, ter clareza das dimensões a serem monitoradas dentro do contexto complexo da cidade é fundamental para uma gestão mais efetiva e possibilitando uma comunicação mais clara entre os atores da cidade.

Nas últimas décadas pode ser verificado uma variedade de modelos de indicadores e rankings de cidades, desde metodologias fechadas até trabalhos científicos com mais detalhamento. As cidades são avaliadas e classificadas segundo suas características econômicas, sociais e geográficas, revelando os melhores e piores locais no que tange qualidade de vida ou condições para atividades econômicas (atratividade para empresas, por exemplo) [GIFFINGER et. al, 2010]. À medida que as cidades investem para obter vantagem competitiva, na economia global do conhecimento há uma necessidade de quantificar, medir, comparar e classificar as cidades com base em seu desempenho. Identificar o posicionamento relativo de uma cidade nos rankings globais revela suas fraquezas e pontos fortes o que colabora para melhorar sua competitividade global. No entanto, os propósitos bastante diferentes e os métodos de análise de dados diferentes dos vários rankings publicados podem produzir rankings bastante variados para as mesmas cidades. [GRANT \& CHUANG, 2012]. O papel dos rankings para as cidades pode ser, por um lado, o de fornecer informações suficientes aos stakeholders (empresas, investidores, cidadãos, outras cidades) mas também ser um guia importante para o desenvolvimento futuro da cidade. As cidades são os centros econômicos (70\% do PIB é gerado pelas cidades) e culturais do mundo cujo progresso depende da gestão efetiva e da elaboração de políticas baseadas em evidências [ISO, 2014c].

Neste contexto, esse trabalho visa analisar quatro modelos de indicadores usados para classificar cidades e permitir monitoramento da evolução das mesmas em direção às cidades sustentáveis, inteligentes e humanas. Serão abordadas as metodologias da ISO 37120, o Índice de Prosperidade das Cidades (em inglês, City Prosperity Index - CPI) da ONU-Habitat, o modelo europeu da Universidade de Viena e o Programa Cidade Sustentável do Brasil.

O restante deste artigo está organizado em mais 6 seções. As seções 2, 3 e 4 expõem o referencial teórico que embasa esta pesquisa. Na seção 5 está descrita a metodologia de análise utilizada e na seção 6 a análise dos resultados. Finalmente, na seção 7 as conclusões e considerações finais.

\title{
SMART CITIES E INDICADORES DE SUSTENTABILIDADE URBANA
}

O estudo sobre o planejamento urbano não é algo novo. O que é novidade é uma conscientização de que os ambientes urbanos terão uma população cada dia maior e que o impacto do ambiente urbano na 


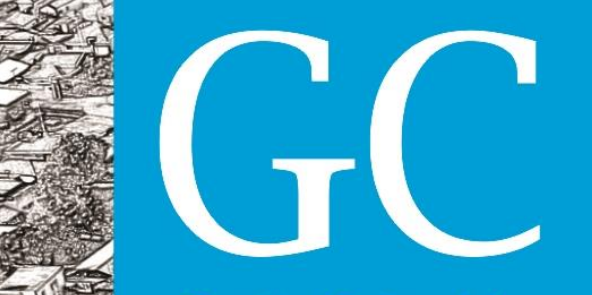

Revista Nacional de
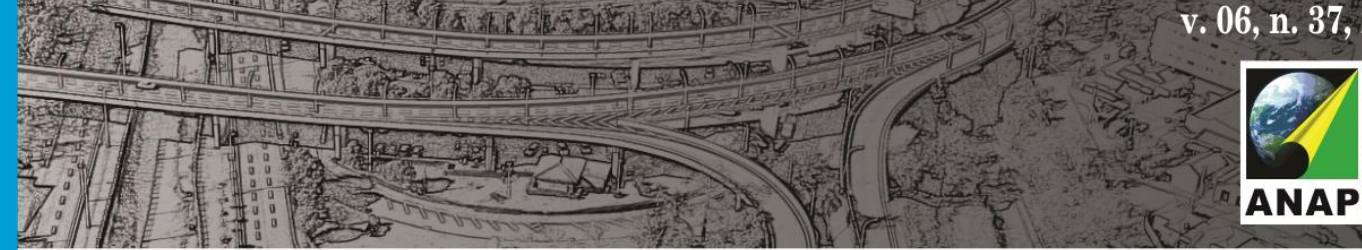

Gerenciamento de Cidades

sustentabilidade do planeta é crucial. As preocupações com os problemas oriundos da urbanização e o uso de tecnologia para resolvê-los também não é tema recente [TOKMAKOFF \& BILLINGTON, 1994], há, entretanto, um crescimento exponencial das pesquisas relacionadas a Smart Cities (Cidades Inteligentes) nos últimos 5 anos devido a disseminação da tecnologia móvel com a ampliação da cobertura da internet, a constatação do crescimento vertiginoso das dimensões da cidade e a busca por sustentabilidade embasada pela preocupação com a questão ambiental [DAMERI, R. P., \& COCCHIA,2013; DAMERI, 2017].

As cidades são sistemas complexos e dinâmicos cuja regulação ou desenvolvimento através do planejamento requer métodos cada vez mais sofisticados para compreender, modelar, prever e formular estratégias e planos para o futuro. $O$ processo de planejamento é suportado por fatos e evidências históricas e atuais, com dados de diversas fontes e tipos que facilitam a tomada de decisão [GEERTMAN et. al, 2017]. Os indicadores de sustentabilidade urbana são ferramentas para o planejamento das cidades e formulação de políticas. Eles devem permitir avaliar o impacto socioeconômico e ambiental de projetos urbanos além de permitir que as cidades possam monitorar o sucesso e o impacto das intervenções de sustentabilidade, [COMISSÃO EUROPEIA, 2015].

[LLACUNA et. al, 2015] apresentam um resumo histórico sobre a promoção de indicadores urbanos de sustentabilidade. A ECO 92 representou um grande marco no monitoramento urbano, onde foi destacado o papel das cidades no caminho da sustentabilidade, seguindo pela Carta de Aalborg que foi o resultado da primeira conferência de sustentabilidade das cidades europeias, ocorrida em 1994. Em 1995, mais de 1200 cidades em todo o mundo ratificaram a carta. O principal acordo alcançado pelos signatários foi elaborar sua própria Agenda Local 21, que consiste em um conjunto de indicadores para monitorar a sustentabilidade. Desde 2006, no entanto, o monitoramento da sustentabilidade diminuiu consideravelmente, e a partir de 2005 indicadores de qualidade de vida obtiveram o foco de empresas privadas como Mercer e The Economist, que publicam relatórios de qualidade de vida das maiores cidades do mundo anualmente. [LLACUNA et. al, 2015] indicam que por volta de 2009 o conceito de Smart Cities começou a ser globalmente reconhecido como o objetivo para qualquer cidade, independentemente do tamanho. Usando como base as experiências anteriores de medir cidades ambientalmente amigáveis e habitáveis, abrangendo os conceitos de sustentabilidade e qualidade de vida, mas com a importante e significativa adição de componentes de TIC (Tecnologia da Comunicação e Informação). Pesquisadores concordam que a sustentabilidade depende dos fatores sociais, econômicos, ambientais e de governança; por exemplo, a produtividade econômica depende de cidadãos saudáveis e felizes, que precisam de fácil acesso à educação, saúde, segurança, alimentação, água, transporte, ar limpo e eletricidade. [COMISSÃO EUROPEIA, 2015].

Segundo [KITCHIN et. al, 2015], os indicadores urbanos podem ser classificados em dois grupos de acordo com seu racional e de como são calculados: Individuais e Compostos. Indicadores individuais se referem a um único fenômeno, por exemplo, o número total de desempregados ou a taxa de desemprego. São medidas geralmente diretas bem definidas e inequívocas e possuem forte representatividade (eles medem o que declaram medir). Em alguns casos eles podem ter natureza indireta quando o fenômeno de interesse é intangível ou não observável, como por exemplo o número de patentes pode ser usado no cálculo do índice de inovação de uma região. Indicadores individuais devem ser objetivos, neutros, independentes de influência externa, rastreáveis ao longo do tempo, sensíveis à mudança, verificáveis e replicáveis, fáceis de interpretar, produzidos e divulgados regularmente. 


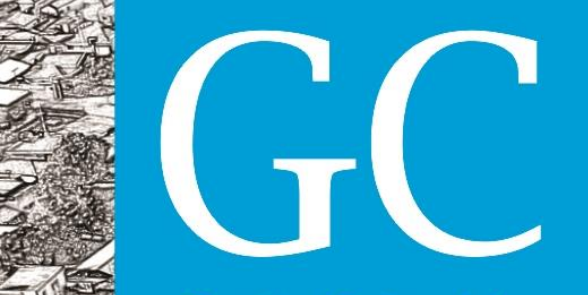

Revista Nacional de
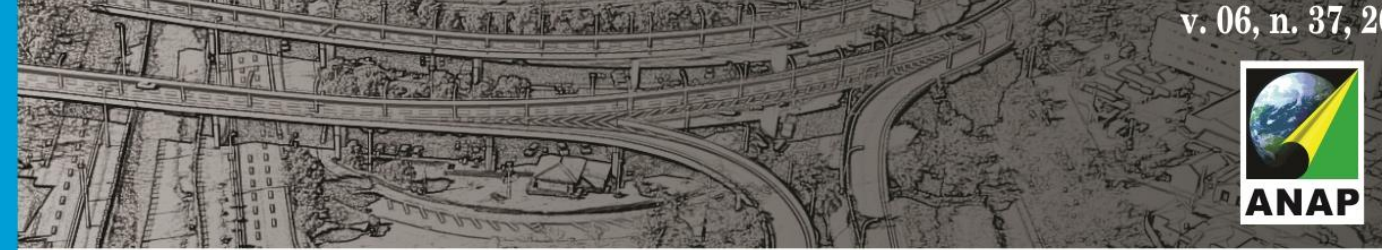

Gerenciamento de Cidades

Os Indicadores Compostos combinam métricas individuais através de aplicação de estatística e sistemas de peso e possibilitam assim uma visão integrada, como por exemplo o custo de reciclagem por tonelada de resíduo que é um indicador composto simples, mas que integra considerações econômicas e ambientais [MACLAREN, 1996]. O índice de desenvolvimento humano é um exemplo de indicador composto pois combina várias métricas como renda, emprego, saúde, educação, bem-estar, acesso a serviços, etc. Outro exemplo são as classificações geo-demográficas que combinam vários indicadores para determinar as características dominantes ao longo do espaço geográfico [KITCHIN et. al, 2015].

\section{RANKINGS DE CIDADES}

Para medir o desempenho de uma cidade em um ranking, o atributo de classificação precisa ser decomposto em indicadores [MEIJERINGA et. al, 2014]. Por exemplo, para medir a sustentabilidade ambiental de uma cidade, indicadores sobre a qualidade do ar (por exemplo, média diária anual de emissões de carbono), consumo de energia (por exemplo, consumo anual de energia em gigajoules por residente) e produção de resíduos (por exemplo, desperdício anual coletado em quilogramas por residente) poderiam ser usados.

Os rankings de cidades produzidos por governos, organizações e institutos de pesquisa têm muitas vezes o objetivo de entender a correlação entre cidades bem-sucedidas e seus atributos fundamentais e capacidade de sucesso econômico, sendo uma ferramenta importante para análise de políticas e sendo úteis para vários atores como políticos, administradores municipais e regionais, empresas e profissionais da comunicação [GRANT \& CHUANG, 2012]. Desta forma, é compreensível a proliferação de rankings produzidos por várias entidades na última década. Entretanto há que se atentar para os aspectos negativos, como descrito por [GIFFINGER et. al, 2010]: rankings de cidades são excessivamente aclamados pelos "vencedores", a fim de melhorar sua imagem pública, enquanto os "perdedores" tendem a ignorar quaisquer resultados.

Outra grande desvantagem da maioria das classificações reside na sua abordagem generalista: a maioria das classificações visa encontrar a cidade "melhor" ou "mais atraente" em termos gerais. Neste intuito, esses estudos tentam cobrir todos os campos da atratividade local, ignorando o fato de que diferentes atividades precisam de condições diferentes. Por exemplo, uma cidade que oferece uma alta qualidade de vida não precisa necessariamente ser a localização ideal para todos os ramos industriais. Da mesma forma, o tamanho da cidade nem sempre é fator determinante nos rankings. A competitividade no desenvolvimento urbano depende dos caminhos históricos da cidade e da capacidade recente de elaborar uma estratégia de desenvolvimento urbano proativa e efetiva, promovendo a inovação ou criatividade em termos culturais, sociais e econômicos. Essa capacidade de enfrentar os respectivos desafios, no entanto, não é necessariamente uma função do tamanho, porque mesmo as cidades de médio ou pequeno porte podem ter essa habilidade [GIFFINGER et. al, 2010].

Dada a variedade de índices de classificação de cidades disponíveis atualmente, há várias perspectivas sobre como as cidades são classificadas, vistas e avaliadas por empresas externas, acadêmicos, líderes políticos e pessoas. É necessário compreender a origem do índice e evitar questões duvidosas, como índices que não detalham a metodologia usada, em especial nos casos onde as cidades vencedoras serão beneficiadas com programas de investimento [GRANT \& CHUANG, 2012]. 


\section{MODELOS DAS CIDADES INTELIGENTES E SUSTENTÁVEIS}

Através de busca exploratória procurou-se por modelos de indicadores para cidades inteligentes e sustentáveis já existentes ou em implementação. Foram selecionados 4 casos:

- ISO 20130

- Índice de Prosperidade das Cidades da ONU-Habitat

- Modelo Europeu da Universidade de Viena

- Programa Cidades Sustentáveis do Brasil

No caso do Brasil também foi revisada a plataforma de dados das cidades do IBGE.

\section{IBGE Cidades}

O sistema "Brasil em Síntese" agrega informações do IBGE sobre os municípios e estados do Brasil, onde são disponibilizadas várias pesquisas do IBGE, infográficos e mapas [IBGE, 2018a]. O sistema permite a comparação das cidades dentro das pesquisas desenvolvidas pelo instituto, como pode ser visto na Figura 1, onde é visualizado o Índice de Desenvolvimento da Educação Básica para o município Aral Moreira comparando o mesmo aos municípios do estado do Mato Grosso do Sul e do Brasil.

Figura 1 - Exemplo do uso do portal de cidades do IBGE

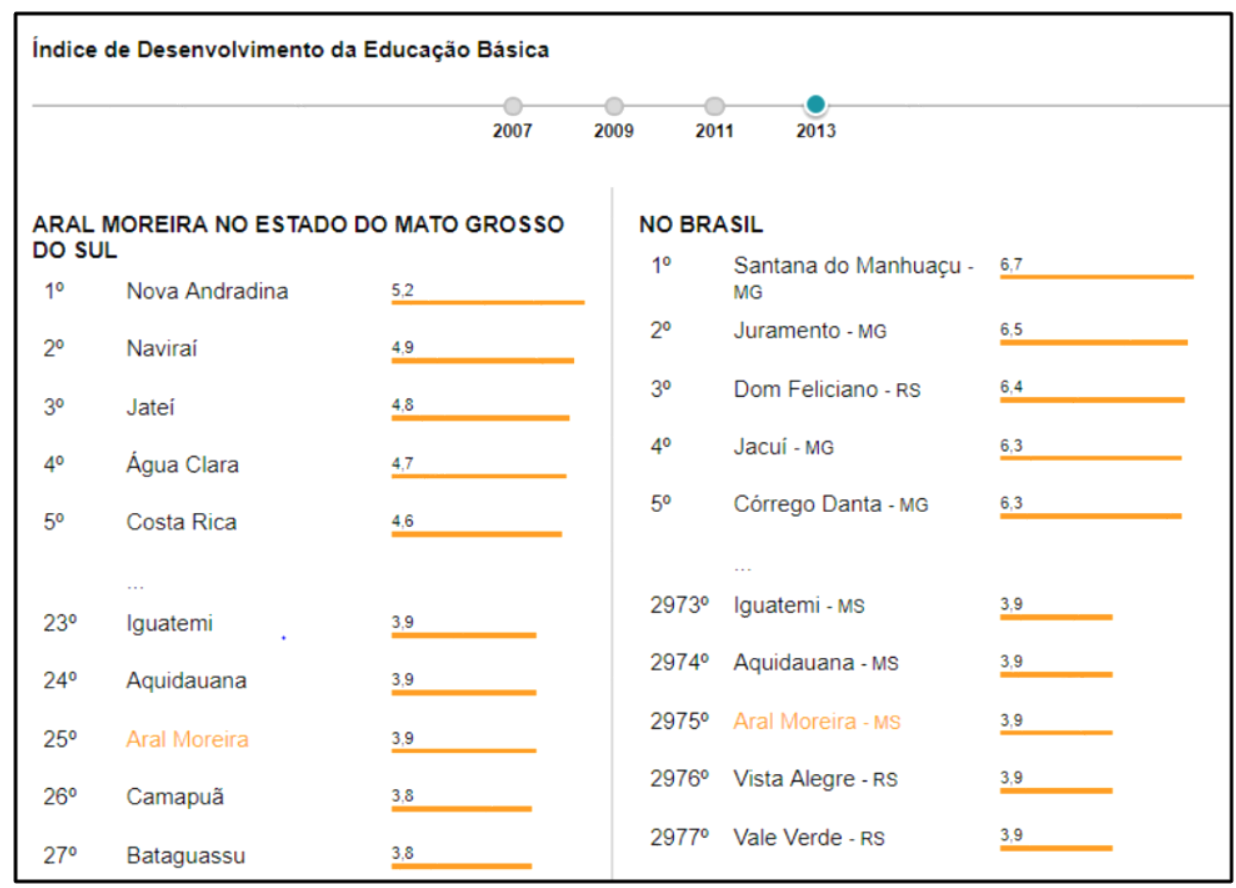

Extraído de [IBGE, 2018a] 


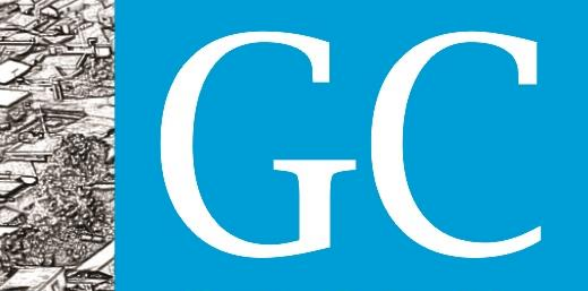

Revista Nacional de

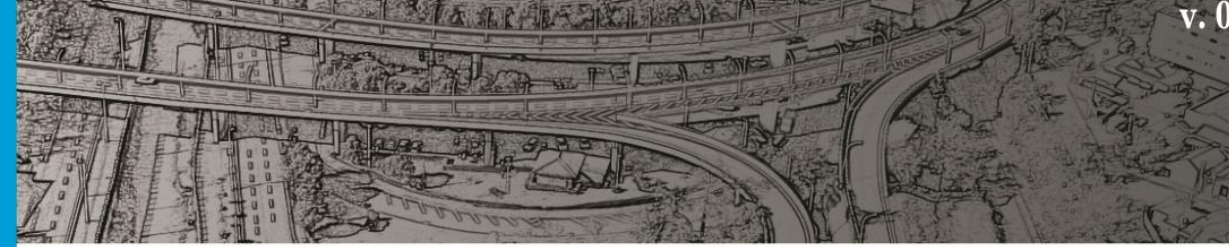

apoio, que pode ser verificado no Anexo I. O padrão fornece também uma linguagem comum para comunicação dos indicadores.

O WCCD (World Council on City Data, Conselho Mundial de Dados da Cidade em português) foi criado para facilitar a adoção e implementação do ISO 37120 para cidades em todo o mundo através de uma plataforma para verificação e comparação de cidades certificadas pela ISO 31720 [WCCD, 2017]. Disponibiliza uma estrutura comum para métricas urbanas o que promove o aprendizado de cidade para cidade, permitindo o melhor desempenho das cidades com o objetivo de melhorar a qualidade de vida geral para cidadãos [MCCARNEY, 2015].

No Brasil a iniciativa Rede Brasileira de Cidades Inteligentes e Humanas (RBCIH) se dedica à criação do Índice Brasileiro de Cidades Inteligentes e Humanas e o Selo Certificador, com indicadores que reflitam se o município está seguindo o passo a passo do rol de ações a serem implementadas, utilizando-se como base a ISO 37120 [RBCIH, 2016].

\section{Modelo CPI da ONU-Habitat}

A ONU-Habitat é um programa das Nações Unidas que possui como missão promover o desenvolvimento de comunidades social e ambientalmente sustentáveis, tendo habitação adequada para todos. Em 2012, a ONUHabitat criou uma ferramenta para medir a sustentabilidade das cidades e auxiliar no acompanhamento do cumprimento do objetivo 11 da agenda de desenvolvimento sustentável para 2030: tornar as cidades e as comunidades inclusivos, seguros, resilientes e sustentáveis. Esta ferramenta conhecida como City Prosperity Index (CPI - Índice de Prosperidade da Cidade) foi acompanhada por uma matriz conceitual, o Círculo da Prosperidade Urbana que é construída através de uma pesquisa qualitativa baseada na percepção do desenvolvimento sustentável da cidade. Essas pesquisas de percepção proporcionam uma oportunidade para as autoridades locais se conectarem e interagirem com cidadãos e especialistas urbanos. A prosperidade implica sucesso, riqueza, condições prósperas, bem-estar, confiança no futuro e oportunidades para todos. Além disso, cidades prósperas oferecem uma profusão de bens públicos, permitindo o acesso equitativo e o desenvolvimento de políticas sustentáveis [CPI, 2017]. A prosperidade, tal como definida por ONU-Habitat, possui uma noção mais ampla e abrangente que tem a ver com políticas claras e um desenvolvimento equilibrado e harmonioso em um ambiente justo.

A ONU-Habitat transformou o CPI em uma iniciativa global conhecida como Iniciativa de Prosperidade da Cidade, que é um diálogo sobre métricas e políticas, oferecendo às cidades de países desenvolvidos e em desenvolvimento a possibilidade de criar indicadores e informações de base, além de definir metas e objetivos que possam apoiar a formulação de políticas baseadas em evidências, incluindo a definição de visões e planos de longo prazo ambiciosos e mensuráveis [CPI, 2015]. O CPI vai de 0 a 100, onde o valor máximo se refere a um nível de prosperidade sólido. $O$ índice geral é calculado a partir de 6 temas com 22 dimensões, que podem ser verificadas no Anexo II. 
comparação efetuadas em [GIFFINGER et. al, 2010], os modelos selecionados foram avaliados considerando os seguintes fatores: Objetivo, Escopo, Metodologia e Disseminação.

\section{ANÁLISE DOS MODELOS E RESULTADOS}

A atividade de análise e comparação dos modelos exige a seleção de um modelo como ponto de partida para a comparação, o que pode ser feito para todos os modelos. Nesta pesquisa partiu-se do modelo europeu por este possuir um número pequeno de áreas chave propiciando uma simplificação de modelos com maior número de temas como PCN e ISO, bem como por ter uma abordagem mais focada no conceito de cidades inteligentes e sustentáveis abordado por esta pesquisa. Foram analisadas todos os temas dos demais modelos classificando os mesmas dentro das 6 áreas chave do modelo europeu: Smart Economy, Smart People, Smart Governance, Smart Mobility, Smart Environment e Smart Living.

No Quadro 1 é possível verificar a classificação através de um conjunto de cores: quando o tema de um modelo se enquadra em mais de uma área chave do modelo europeu a cor principal corresponde ao tema mais aderente e ao lado está uma segunda cor representando um tema que também se enquadra a referida área chave do modelo europeu, entretanto menos aderente. Por exemplo no modelo CPI a dimensão Infraestrutura tem aderência com duas áreas chave: Smart Mobility e Smart Living, nesta ordem de aderência.

Quadro 1 - Relações das dimensões das metodologias

\begin{tabular}{|c|c|c|c|}
\hline \multicolumn{2}{|l|}{ Modelo Europeu } & Modelo City Prosperity Index (CPI) & \multirow[b]{3}{*}{ (SL) } \\
\hline SMART ECONOMY & SE & Produtividade (SE) & \\
\hline SMART PEOPLE & SP & Infraestrutura (SM) & \\
\hline SMART GOVERNANCE & SG & Qualidade de Vida (SP) & \multirow[t]{4}{*}{ (SL) } \\
\hline SMART MOBILITY & SM & Equidade e Inclusão social (SL) & \\
\hline SMART ENVIROMENT & SE & Sustentabilidade ambiental (SE) & \\
\hline SMART LIVING & SL & Governança e Legislaçăo (SG) & \\
\hline \multicolumn{2}{|l|}{ Modelo ISO 37120} & \multicolumn{2}{|l|}{ Modelo Programa Cidades Sustentáveis (PCS) } \\
\hline \multicolumn{2}{|l|}{ Economia (SE) } & \multicolumn{2}{|l|}{ Ação Local para a Saúde (SL) } \\
\hline \multicolumn{2}{|l|}{ Educação (SP) } & \multicolumn{2}{|l|}{ Bens Naturais Comuns (SE) } \\
\hline \multicolumn{2}{|l|}{ Energia (SE) } & \multicolumn{2}{|l|}{ Consumo Responsável e Opçöes de Estilo de Vida (SE) } \\
\hline \multicolumn{2}{|l|}{ Meio Ambiente (SE) } & Cultura para a Sustentabilidade (SL) & \multirow{2}{*}{$\begin{array}{l}\text { (SP) } \\
\text { (SL) }\end{array}$} \\
\hline \multicolumn{2}{|l|}{ Finanças } & Do Local para o Global (SE) & \\
\hline \multicolumn{2}{|l|}{ Fogo e Resposta a Desastres (SL) } & Economia Local Dinâmica, Criativa e Sustentável (SE) & \\
\hline \multicolumn{2}{|l|}{ Governança (SG) } & Educação $p /$ Sustentabilidade e Qualidade de Vida (SP) & \\
\hline \multicolumn{2}{|l|}{ Saúde (SL) } & Equidade, Justiça Social e Cultura de Paz (SL) & \\
\hline \multirow{2}{*}{\multicolumn{2}{|c|}{$\begin{array}{l}\text { Lazer (SL) } \\
\text { Segurança (SL) }\end{array}$}} & Gestão Local para a Sustentabilidade (SE) & \\
\hline & & Governança (SG) & \\
\hline \multicolumn{2}{|l|}{ Habitaçăo (SL) } & Melhor Mobilidade, Menos Tráfego (SM) & \\
\hline \multicolumn{2}{|l|}{$\begin{array}{l}\text { Resíduos Sólidos (SE) } \\
\text { Telecomunicaçōes e Inovaçōes (SM) }\end{array}$} & Planejamento e Desenho Urbano (SG) & (SL) \\
\hline \multicolumn{4}{|l|}{ Transportes (SM) } \\
\hline Planejamento Urbano (SG) & (SL) & & \\
\hline \multicolumn{4}{|l|}{ Aguas Residuais (SE) } \\
\hline Agua e Saneamento (SE) & & & \\
\hline
\end{tabular}

Fonte: Elaborado pelos autores 


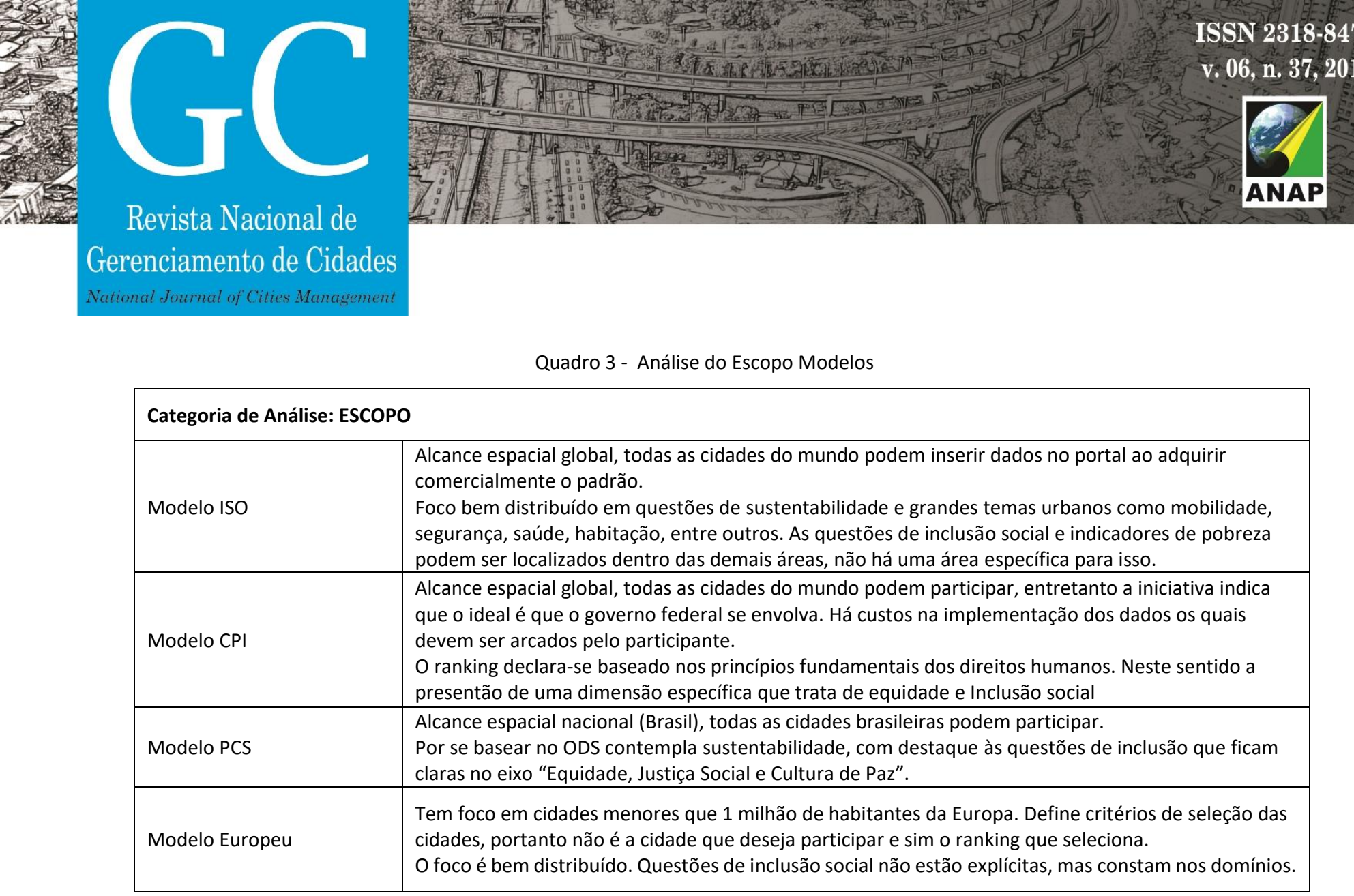

Os modelos CPI e PCS estão vinculados aos ODS, sendo que CPI foca no objetivo 11 e PCS trata de todos os 17 objetivos, o que leva este modelo a um número alto de indicadores. No Quadro 4, fica claro que, em relação a classificação e comparação das cidades, somente PCS não se utiliza de padronização e peso dos indicadores no momento da avaliação desta pesquisa. Desta forma, PCS não calcula um índice geral para as cidades, busca, entretanto, uma comparação para cada indicador e, portanto, não deve ser classificado como um ranking. Seria interessante criar uma padronização para tornar mais fácil a comparação das cidades brasileiras em cada tema.

Todos os modelos usam os mesmos conjuntos de indicadores independentemente do tamanho da cidade à exceção do modelo europeu, o qual restringe seu escopo às cidades entre 100 mil e 1 milhão de habitantes e possui mais de um modelo disponível. 


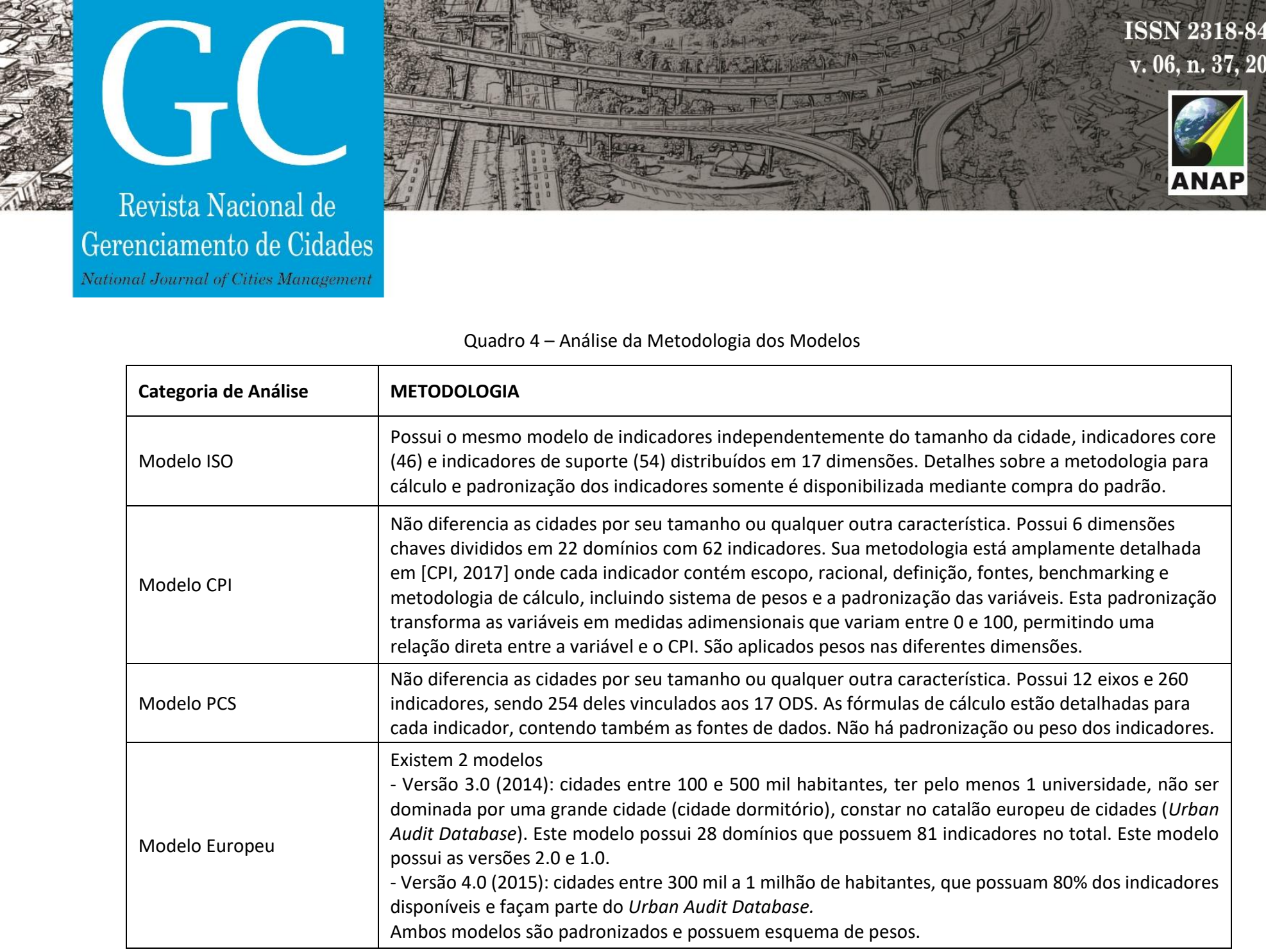

Com exceção do modelo Europeu, que tem critérios para seleção das cidades que independem do desejo de participar do município, a participação nos demais modelos é viável a qualquer cidade dentro do escopo espacial (no caso do PCS: qualquer cidade do Brasil). A que se atentar que alguns dos modelos demandam mais ou menos custos ao gestor local, com investimentos mínimos para organizar o framework de obtenção dos dados ou compra da metodologia como ocorre no modelo ISO, que exige a aquisição comercial do padrão como ponto de partida ou CPI que chega a mencionar custos médios de USD 150 mil por município. Talvez este fato seja causador do baixo número de cidades participantes destes modelos globais como pode ser verificado o Quadro 5. Já no caso do PCS, o grande número de indicadores aliado a uma baixa capacidade de obter estes dados pode ser a razão de apenas 79 cidades constarem no banco de dados geral do sistema no momento desta pesquisa, correspondendo a menos de 1,5\% dos municípios brasileiros.

Todos os modelos possuem websites com opções para geração de gráficos, mapas e outras ferramentas que permitem a comparação entre duas ou mais cidades. Dos 4 modelos somente CPI e PCS permitem a extração da base completa de cidades e indicadores. 
